\title{
Virus Taxonomy, Sequence Relationships and Evolution
}

The evolution of viruses is a subject which has been discussed at several international congresses of virology. These discussions lent heavily on information derived from ser-ological experiments, and the classification of viruses in turn has placed great reliance on the relationships derived from the same information. The fact that serological results are themselves based on amino acid sequences coded for by only a small portion of the virus genome clearly restricts their value. Now, however, with the increasing availability of gene sequences, the relationships between viruses can be determined with more certainty. This development makes discussions of virus evolution more meaningful and it seems likely that better schemes for classifying viruses may emerge from such information.
A. At the 1987 congress in Edmonton
the papers presented in a symposium on virus taxonomy sequence relationships and evolution
organized for the International Committee on the Taxonomy of Viruses by B.M. Gorman and F. Brown underlined the impact which gene sequencing is having on these studies. Five of the papers presented at the symposium are included in this issue of Intervirology.

F. Brown B.M. Gorman 